\title{
Business Process Improvement in Abnoba
}

\author{
Konstantin Hoesch-Klohe and Aditya Ghose \\ Decision Systems Lab (DSL), \\ School of Computer Science and Software Engineering, \\ University of Wollongong
}

\begin{abstract}
A key element of any approach to meeting the climate change challenge is the ability to improve operational efficiency in a pervasive fashion. The notion of a business process is a particularly useful unit of analysis in this context. In the Abnoba framework, we enable business process management (and in particular, business process design/redesign) with explicit support for the environmental sustainability aspects of processes. This article extends our earlier work on the framework by introducing and elaborating a machinery for (semi-)automated process re-design discovery. The machinery leverages a library of process snippets/fragments, used to replace fragments from the library with fragments of the process design, such that the process re-designs meets functional-, process provisioning-, and compliance requirements and the sustainability profile is improved.
\end{abstract}

Keywords: automated process improvement, process provisioning, semantic annotation, green/sustainable BPM, Abnoba.

\section{Introduction}

A key element of any approach to meeting the climate change challenge is the ability to improve operational efficiency in a pervasive fashion. The notion of a business process is a particularly useful unit of analysis in this context. In the Abnoba framework, we enable business process management (and in particular, business process design/re-design) with explicit support for the environmental sustainability aspects of processes. This article extends our earlier work on the framework [123. by elaborating a machinery for (semi-)automated process improvement, using a library of semantic annotated process snippets. Modeling organizational behavior in a business process we are interested in finding process design alternatives, which constitute an improvement, with respect to certain measures (e.g. CO2 emission), over the as-is design. Various techniques have been described in the literature for process improvement, see Reijers and Mansar 4 for a survey. While existing work primarily provides process re-design guidelines to the analyst, less attention is given to automated process re-design/improvement machineries. Existing machineries for automated process improvement (e.g. [5]) mainly focus on parallelizing activities. While parallelizing activities can have a positive impact on the process cycle time, the resulting process re-design is not necessarily superior with respect to other criteria like the sustainability profile. 
Furthermore, the number of applicable process re-designs is limited by the size of the search space constituting all possible process re-designs, which can be devised by parallelizing activities of the process design under consideration. By extending the size of the search space, we can increase our chances to find more preferable process re-designs. We extend the search space by devising a library of process snippets, which constitute best practice process designs and fragments for a given domain. Essentially, we seek to replace process fragments of an as-is process design with other fragments (drawn from the library) in a manner that ensures that the original goals of the process are still realized (the same desired functional outcome is achieved), but the sustainability profile of the resulting process design is improved. Such a machinery requires the process designs (and fragments in the library) to be annotated with semantic effects and a machinery for effect accumulation. For this, we build upon of the ProcessSEER framework 6 6 7 /8. However, when introducing new process fragments (from the library) to the as-is process design we must also ensure that the process re-design, can still be executed in the organizational resource context. In this context, we describe a rich resource model, a machinery for correlating resources with process designs and finally the resulting concept of (abstract and concrete) process provisioning. These results extend and improve upon earlier work on resource modeling 910 1112 13. In addition, it must be ensured that the process re-design does not violate any compliance requirements. Business process compliance for semantic annotated process designs can be found in [7]6], we omit details for brevity. In the remaining article we first (Section 2) describe work done on resource modeling and introduce the concept of process provisioning, before (in Section 4) we elaborate the proposed machinery for process improvement and finally conclude (in Section 5) the article.

\section{$2 \quad$ Resource Modelling}

In this following we extend our previous work on resource modeling [2], by distinguishing between different resource and relationship types. In addition to a "Use" relationship (denoting that one resource is used by another), we allow relationships of type "IS-A" and "Whole-Part", which enables us talk about resources on different level of abstraction. Furthermore, a resource can be of type "schedulable resource class", "unschedulable resource class", and "resource instance. Both the new resource- and relationship types are taken from Podorozhny et al. [9], who introduces and applies a resource management system in agent and activity coordination. The new relationship and resource types are described in more detail as follows:

- Resource Types: A resource instance is a unique representation of an entity from the physical environment of discourse. It therefore denotes a specific resource in the organization. A schedulable resource class is a set of resources, which are substitutable for each other and can be allocated to an activity. An unschedulable resource class is used to structure resources with 
similar properties that are not schedulable. We use this class to describe resources like electricity, which cannot directly be used by an activity, but are rather indirectly via electrical devises.

- Relationship Types: A IS-A relation is used to denote that entities can share a set of attributes, where the attributes of one entity are inherited by all specializations for that entity. The Whole-part relation is another abstraction mechanism, where the linked resources constitute part of an aggregated resource. The Use relation (Podorozhny et al. uses the term "requires relation") denotes that a resource entity requires another entity to be used.

We formally define this model as follows: A resource model $R M$ is denoted by a labeled digraph $\left\langle V, E, \Omega_{V}, \Omega_{p} \Omega_{E}, f_{V}, f_{E}, f_{p}\right\rangle$

- $V$ is an partially ordered set of vertices $v \in V$.

- $E$ is a set of edges such that $(x, y) \in E \subseteq V \times V$, where $x, y \in V$ and $x$ is pointing to $y$.

- $\Omega_{p}$ is a set of properties, denoted by a triple $\langle i d$, type, value $\rangle$, such that $i d$ is the unique identification of a property, type denotes the type of property, and value is a set of values.

$-\Omega_{V}$ is a set of vertex labels where each label is a tuple $\langle i d$, type $\rangle$, such that $i d$ is the unique identification of the label and type denotes the type of resource where type $\in$ unschedulable resource class, schedulable resource class, resource instance;

$-f_{p}: \Omega_{V} \rightarrow 2^{\Omega_{p}}$ is a function mapping labels to elements in $\Omega_{p}$.

$-\Omega_{E}$ is a set of edge labels denoted by $\langle i d$, type $\rangle$, such that $i d$ is the unique identification of an edge label; type is the type of the edge where type $\in$ \{IS-

A, Whole-Part, Use\};

$-f_{V}: V \rightarrow \Omega_{V}$ is a function mapping vertices to a vertex label.

$-f_{E}: E \rightarrow \Omega_{E}$ is a function mapping edges to an edge label.

In addition the following rules over the model must hold. (1) Subgraphs of $R M$, where all edges are of type "IS-A" must be a tree. (2) There exist no two edges of type "IS-A" and "Whole-Part" between the same two nodes.

\section{Process Provisioning}

We refer to the correlation of resource models and process designs as the provision of the process design by the resource model. We consider process provisioning in two different ways. In abstract process provisioning we correlate an activity in the process design with a resource in the resource model. The resource in question might be arbitrarily abstract, e.g. a "printer" resource, or a more specific "inkjet printer" resource, or an even more specific "inkjet printer model x of manufacturer y" resource. By establishing an abstract provisioning relation between a task in a process design (say "print report") and any of these resources we merely assert a provisioning relation (e.g. the "print report" activity shall use the "inkjet printer" resource, or an even more specific resource). In concrete 
process provisioning, we annotate the abstract provisioning relation with specific quality of service (QoS) requirements. For instance, we might establish a concrete provisioning relation between the "print report" activity and an "inkjet printer" resource which admits a configurable image resolution setting by stating the minimum image resolution requirements.

Technically, we correlate process designs with resource models by annotating each activity of a process design with an expression. The annotated expression references one or more resource entities, where each reference is accompanied with a (potentially empty) set of QoS requirements. Each QoS requirement is described by a triple $\langle Q o S$, value, 1$\rangle$, where $Q \circ S$ denotes the QoS measure of consideration, value the required value, and 1 the most preferred value (as in the algebraic c-semiring structure 14, which we use in [3] to handle multidimensional qualitative and quantitative measures). The existence of a resource model permits us to explore a range of provisioning options. An activity can be provisioned by by a set of resources $R$ if (1) it requires $R^{\prime}$ resources and all elements of $R^{\prime}$ are also an element of $R$ or of an specialization in $R$. (2) $R^{\prime}$ meets all QoS requirements stated in the provisioning relation. A resource meets a QoS requirement if the QoS value is equal or between the required value and the most preferred value (1). A process design can be provisioned if all its activities can be provisioned.

It can be observed that the provisioning relations serve as constraints, restricting the space of applicable process designs and applicable resource models. This is particular important in the face of process re-design and optimization, where it must be ensured that the process re-design can still be provisioned by the resource context. Similar, for any changes on the resource context (e.g. exchanging resources, or deploying a process design in a new resource environment) provisioning must be ensured. In the following we will focus on process design improvement, omitting resource optimization, which we seek to address in future work.

\section{Business Process Improvement}

Process improvement must involve process re-design to obtain processes that achieve the same (functional) goals, while minimizing the environmental impact (and potentially other non-functional criteria as well). To achieve this, we need (1) the ability to annotate process designs with detailed specifications of functional effects (2) the ability to correlated organizational goals with process designs (3) the ability to search for process re-designs through a space of alternative process designs.

\subsection{Preliminaries}

This subsection provides the preliminaries on annotating and accumulating functional effects, correlating goal models with process models, and change patterns for design time change on process designs. 
Annotating and Accumulating Functional-Effects. A process design can be semantically enriched by specifying the outcome of each activity. This is done by annotating each activity with its immediate effects (post conditions), denoting the consequence of executing the respective activity in some state in the environment. Immediate effects are represented as logic statements in conjunctive normal form. We pairwise accumulate effects to receive a cumulative effect. Pairwise accumulation is done by set union the effects of two sequentially linked activities, such that the combined set of effects is consistent (we can view sentences in conjunctive normal form as sets of clauses, without loss of generality). If the resulting set is not consistent the cumulative effects consists of the effects of the second activity and as much effects of the first activity as can be consistently included (effects are overwritten/undone by the second activity). Furthermore, all cumulative effects must be consistent with a background knowledge base (KB) containing rules (e.g. for compliance). Note that this KB can also be used to state pre-conditions (conditions that must hold before the activity can be executed) in a centralized manner. Effect accumulation in parallel environments is done by pairwise accumulating the cumulative effects of each branch with the effects of the activity directly following the AND-join and combining these sets via set union. A detailed description of this machinery can be found in 678 . The functional outcomes of a process are its cumulative effects at the end-event. A process design can have multiple cumulative effects outcomes one for each distinct execution path.

Correlating Goals with Process Models. A goal is an objective the organization seeks to achieve. It can be formulated at different levels of abstraction ranging from abstract goals (e.g. strategies) to further decomposed goals. A rich body of knowledge on goal modeling can be found in the goal-oriented requirements engineering literature. We list besides others [15 16]. Correlating process models with goal models is crucial to ensure that the process environment, being under change, maintains to satisfy the organizational objectives. Koliadis and Ghose 17 proposes the GoalBPM methodology for relating business process models (in BPMN) to functional goals (in KAOS [16]) via satisfiability links. These links are drawn between the process model and goals of the goal model, denoting the goals satisfied by the process design. A goal is represented as logic statement and is in its simplest form a single literal $\alpha$, or conjunction of literals $\{\alpha \wedge \beta\}$, for example "package send" and "bill send".

A process design satisfies the organizational objectives if it satisfies all correlated goals. A correlated goal is satisfied by a process design if all its cumulative effects at the end-event entail the goal. For example the cumulative effect $\{\alpha \wedge \beta \wedge \gamma\}$ entails (denoted by $=$ ) the goal $\{\alpha \wedge \beta\}$ ). The implication of requiring all cumulative effects to entail a goal is that each path through the process design must lead to goal satisfaction. It is obvious that a path, which does not satisfy the objectives of a process is superfluous. Note that a correlated goal does not have to denote "positive" outcomes only. For example, a process "credit card application" might have the goal "application processed" and the background 
$K B$ tells us that either the cumulative effect "credit card issued" or "credit card declined" satisfies the goal.

Process Change. Weber et al. 18] introduce high-level change patterns and change support features to assess existing process aware information systems in their ability to deal with process change. The high-level change patterns are divided into patterns supporting structural process adaption (adaption patterns) and patterns for built-in flexibility at predefined regions (e.g. to add information during run-time). We focus on the adaption patterns, since our focus is on design time optimization. Equally to Weber et al., we use the term process fragment to denote atomic activities, hammocks (sub-process graphs with a single entry and exit point), and complex activities (sub-process). The change patterns delete process fragment from process design and replace process fragment by another fragment, are of interest to us. Applying these change patterns on a process design we are able to discover potential process re-designs. Due to the nature of process fragment (single entry and exit point) the well-formedness of the process re-design is ensured.

\subsection{A Machinery for Process Improvement}

In this subsection we build on the preliminaries of the previous subsection and introduce a machinery for discovering process re-designs using a library of process fragments. Figure 1 provides an overview of the procedure. The original process design (upper left corner), is (1) disassemble into its process fragments (lower left corner). (2) The change pattern "delete process fragment" is applied to delete potentially obsolete fragments. (3) search for substitutable fragments in the library (lower right corner). (4) Replace substitutable fragments and check whether the resulting process re-design achieves the same desired functional outcome. (5) Order process re-designs according to their environmental profile. (6) Repeat previous steps until termination criteria is met.

Disassemble Process Design. In the first step we disassemble the as-is process design in a straight forward fashion into its process fragments (details omitted for brevity).

Delete Obsolete Fragments. In the second step we identify and delete obsolete fragments, by checking for each identified process fragment, whether it can be deleted, such that the resulting process design still satisfies the correlated goals (desired functionality). We do this by accumulating the semantic effects to get the cumulative effects. We then check whether the cumulative effects entail all correlated goals (as described in subsection 4.1). If the cumulative effects entail the correlated goals the process design is kept and the previous step repeated until no more fragment can be deleted.

Find Substitutable Fragments. In the third step we seek to find a substitutable process fragments, for each remaining process fragment, in a library 


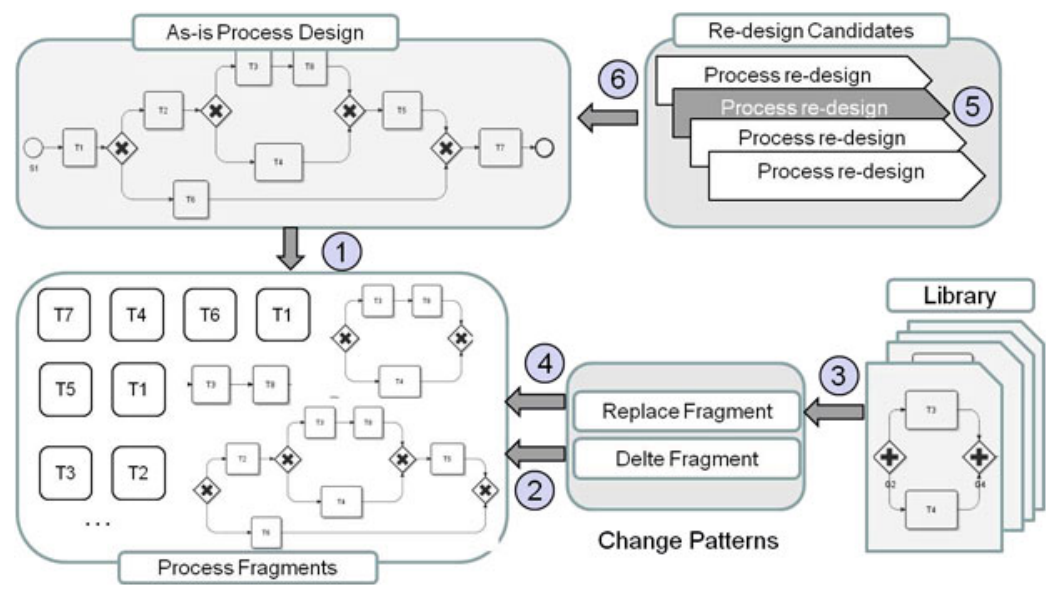

Fig. 1. Process Improvement

of process fragments. The library constitutes as a set of (semantic- and provisioning constraint annotated) process design snippets, denoting "best practice" solutions. The library is not restricted to process snippets, but could also include services (which can be seen as complex activities), derived from a service broker. Searching for replacements in the library can hence be utilized as an instrument for business functionality outsourcing. A process fragment $p^{\prime}$ is potential substitute for another process fragment $p$ if and only if every terminal effect scenario of $p$ is entailed by some terminal effect scenario of $p^{\prime}$. For example, consider a process fragment with two sequentially linked activities $T_{n}$ and $T_{m}$ with a cumulative effect of $\alpha \wedge \beta$ at $T_{m}$. This process fragment is substitutable by an process fragment in the library with one activity $T_{n}$ and the effect $\alpha \wedge \gamma \wedge \beta$, since $\{\alpha \wedge \gamma \wedge \beta\} \models\{\alpha \wedge \beta\}$.

Replace Substitutable Fragments. In the fourth step we replace each process fragment of the as-is design with a substitute in the library (if there exists one). Each such replacement denotes a potential process re-design, such that the number of identified substitutable fragments denotes the number of potential process re-designs. Due to the nature of process fragments the well-formedness of each such process re-design can be guaranteed. However, it is not guaranteed that the potential process re-design candidate has the intended functional (cumulative effect) outcomes and whether it violates any compliance requirements (details omitted for brevity). This is due to the fact that a fragment from the library can unintentionally add additional effects to the process design (e.g. $\gamma$ in the example given above), which might conflict with effects of other activities, resulting in unintended cumulative effects and finally in non-satisfaction of correlated goals.

A process fragment $p^{\prime}$ is defined to be a viable process re-design for another process fragment $p$ of a process design $P$ if and only if: (1) For every cumulative 
effect outcome $s^{\prime}$ in process design $P^{\prime}$ obtained by replacing $p$ with $p^{\prime}$ in $P, s^{\prime} \models$ $G_{p}$, where $G_{p}$ is the goal of process $P$. (2) $P^{\prime}$ does not violate any compliance requirements. (3) $P^{\prime}$ can be provisioned. We use the ProcessSEER machinery, described in subsection 4.1 to get the cumulative effects. Finally, all potential process re-designs that satisfy the correlated goals, can be provisioned, and do not violate any compliance requirements, are added to a set of process re-designs.

Please note that the effect accumulation procedure for parallel environments assumes that the cumulative effects of each branch are consistent - there exists no execution orders of activities in an parallel environment that leads to inconsistency. As pointed out by [8] such a scenario only occurs if the process is designed erroneous. Modeling activities in parallel we implicitly accept that the execution order between activities on different branches does not have an impact on the functional result of the process. However, in our case we cannot guarantee that the assumption still holds. We therefore have to determine all interleaving of a parallel environment to identify execution orders leading to undesired cumulative effects. This can be a computational expensive task for large process models. However, we can reduce the computational costs for most cases by devising a pre-checking procedure that catches potential conflicts. This is done as follows: Let us devise a set $\Omega$ for each branch $n, \ldots, m$ in a parallel environment, where $\Omega_{n}$ is the set of all effect elements of a branch $n$. A potential conflict is identified if $\Omega_{n} \cup \ldots \cup \Omega_{m} \cup K B \models \perp$. We only have to determine the cumulative effect of all possible interleaving of the parallel environment, if there exists a potential conflict. Although, we cannot always exclude this scenario, we note that we are in the game of design time improvement, where time constraints are less strict.

Order Process Re-designs. In the fifth step we order the process re-designs according to their sustainability profile, such that the most preferred process design can be identified. In 3] we leverage the algebraic c-semiring structure 14 to compare "green" QoS values of both qualitative and quantitative nature. Essentially, a comparison operator " $\oplus$ " is defined such that $a \oplus b=a$ if $a$ is more preferred as $b$ ( $a \geq b$, where $\geq$ is a partial order over all values of a measure). The process is straight forward for a single measure and a process re-design with a single execution path. For process designs with multiple execution paths we end up with multiple values for a single measure, one for each path. This is handled as follows. (1) For a QoS measure of quantitative nature we use the average value over all paths. This approach essentially place equal weighting on all paths. However, this approach might be erroneous due to the fact that some rarely executed scenarios might skew the mean value. To avoid this issue, we can consider the probability (if available) of taking a path through the process design during execution. Such figures can be derived from process logs or user experience.(2) For a QoS measure of qualitative nature we either chose the "best" or "worst" value as corresponding value. In cases where we want to compare process re-designs with respect to multiple QoS measures, we compose each QoS measure (denoted by a single c-semiring), where the composite structure is a c-semiring as well (proofed in [14]). Hence we can use the " $\oplus$ " comparison 
operator of the composite structure to compare the values. For a more detailed discussion see [14] and [3].

Repeat Previous Steps. In the sixth step the procedure is repeated for all identified process re-designs until a termination criteria is met (e.g. number of iterations, duration, no improvement after $\mathrm{x}$ amounts of iterations in a row). Please note that the described procedure is not complete in the sense that we cannot guarantee that the best possible process re-design has been identified. Nonetheless, we believe that the procedure can already provide significant improvements after a few iterations. Furthermore, the procedure can be interrupted at any time providing the user with the current order over the already identified process re-design suggestion.

\section{Conclusion}

In this article we elaborated a machinery for process improvement and the concept of process provisioning, being part of the Abnoba framework for green business process management. The process improvement machinery leverages a library of (best practice) process snippets to discover potential process redesigns, while ensuring that functional, compliance, and process provisioning requirements are met. This machinery will allow us to provide tool support for environmental aware semi-automatic process improvement. The described machinery behaves semi-automatic, because it does not consider interconnections (e.g. message flows) across and between pools and hence requires the analyst to correctly place these links. Nevertheless, we believe that the workload for the analyst can be decreased, and that further (potentially overlooked) process re-design alternatives can be revealed.

Future work is concerned with developing a thorough implementation for an industry evaluation. Furthermore, we seek to explore machineries for resource optimization and explore the interactions with the process improvement machinery.

\section{References}

1. Ghose, A., Hoesch-Klohe, K., Hinsche, L., Le, L.S.: Green business process management: A research agenda. Australian Journal of Information Systems 16(2) (2009)

2. Hoesch-Klohe, K., Ghose, A.: Towards Green Business Process Management. In: Proceedings of the 7th International Conference on Services Computing (Industry and Application Track) (2010) (to appear)

3. Hoesch-Klohe, K., Ghose, A.: Carbon-Aware Business Process Design in Abnoba. In: Maglio, P.P., Weske, M., Yang, J., Fantinato, M. (eds.) ICSOC 2010. LNCS, vol. 6470, pp. 551-556. Springer, Heidelberg (2010)

4. Reijers, H., Liman Mansar, S.: Best practices in business process redesign: an overview and qualitative evaluation of successful redesign heuristics. Omega 33(4), 283-306 (2005) 
5. Netjes, M., Reijers, H., Aalst, W.: On the Formal Generation of Process Redesigns. In: Ardagna, D., Mecella, M., Yang, J. (eds.) BPM 2008 Workshop. LNBIP, vol. 17, pp. 224-235. Springer, Heidelberg (2009)

6. Ghose, A., Koliadis, G.: Auditing business process compliance. In: Krämer, B.J., Lin, K.-J., Narasimhan, P. (eds.) ICSOC 2007. LNCS, vol. 4749, pp. 169-180. Springer, Heidelberg (2007)

7. Ghose, A., Koliadis, G.: Pctk: A toolkit for managing business process compliance. In: Proc. of the 2008 International Workshop on Juris-Informatics, JURISIN 2008 (2008)

8. Hinge, K., Ghose, A., Koliadis, G.: Process seer: A tool for semantic effect annotation of business process models. In: IEEE Computer Society Press (ed.) Proc. of the 13th IEEE International EDOC Conference, EDOC 2009 (2009)

9. Podorozhny, R., Lerner, B., Osterweil, L., Podorozhny, R., Lerner, B., Osterweil, L.: Modeling resources for activity coordination and scheduling. In: Proceedings of Coordination 1999, pp. 307-322 (1999)

10. Kwan, M., Balasubramanian, P.: Adding workflow analysis techniques to the IS development toolkit. In: Proceedings of the Hawii International Conference on System Science, vol. 31, pp. 312-321 (1998)

11. Russell, N., Ter Hofstede, A., Edmond, D., van der Aalst, W.: Workflow resource patterns: Identification, representation and tool support. In: Pastor, Ó., Falcão e Cunha, J. (eds.) CAiSE 2005. LNCS, vol. 3520, pp. 13-17. Springer, Heidelberg (2005)

12. Muehlen, M.: Resource modeling in workflow applications. In: Proceedings of the 1999 Workflow Management Conference, pp. 137-153 (1999)

13. Muehlen, M.: Organizational management in workflow applications-issues and perspectives. Information Technology and Management 5(3), 271-291 (2004)

14. Bistarelli, S., Montanari, U., Rossi, F.: Semiring-based constraint satisfaction and optimization. Journal of the ACM (JACM) 44(2), 236 (1997)

15. Yu, E., Mylopoulos, J.: From ER to 'AR'-modelling strategic actor relationships for business process reengineering. International Journal of Cooperative Information Systems 4, 125-144 (1995)

16. Van Lamsweerde, A., et al.: Goal-oriented requirements engineering: A guided tour. In: Proceedings of the 5th IEEE International Symposium on Requirements Engineering, Toronto, Canada, vol. 249, p. 263 (2001)

17. Koliadis, G., Ghose, A.K.: Relating Business Process Models to Goal-Oriented Requirements Models in KAOS. In: Hoffmann, A., Kang, B.-h., Richards, D., Tsumoto, S. (eds.) PKAW 2006. LNCS (LNAI), vol. 4303, pp. 25-39. Springer, Heidelberg (2006)

18. Weber, B., Rinderle, S., Reichert, M.: Change patterns and change support features in process-aware information systems. In: Krogstie, J., Opdahl, A.L., Sindre, G. (eds.) CAiSE 2007 and WES 2007. LNCS, vol. 4495, pp. 574-588. Springer, Heidelberg (2007) 